\title{
Introduction to Dr. Bartolozzi's feature section
}

\author{
Morton A. Meyers \\ Department of Radiology, School of Medicine, Health Sciences Center, State University of New York, Stony Brook, \\ NY 11794-8460, USA
}

This issue's Feature Section is graced by the Guest Editorship of Professor Carlo Bartolozzi who has organized and solicited articles from experts who detail cutting edge advances in MR of the liver along with their clinical applications.

Professor Bartolozzi (Fig. 1) serves for over 20 years as Chair of Radiology at the University of Pisa. His department is particularly notable for both its clinical expertise in all the imaging specialities and its academic productivity. A further testament to his wide-ranging abilities is shown by the fact that he was also Director of the Department of Oncology, Transplants, and Advanced Technologies in Medicine from 1999 to 2007.

He has published a score of monographs and over 250 peer-reviewed papers, particularly dealing with liver imaging, the diagnosis of hepatocellular carcinoma, and its treatment via interventional procedures. He is past president of the European Society of Magnetic Resonance in Medicine and Biology (ESMRMB) and the European Society of Gastrointestinal and Abdominal Radiology (ESGAR), and was recently honored by being awarded its Gold Medal. ABDOMINAL IMAGING gains much from his contributions as a distinguished member of its Editorial Board. On a personal level, Professor Bartolozzi is a warm, engaging, and charming individual.

If the reader wishes to know not only where we are today but also where we likely will be tomorrow regarding MR of the liver, this Feature Section is the source. Comprehensive yet readable, theory-based yet practical, focused yet predictive, it inspires as it is realistic.

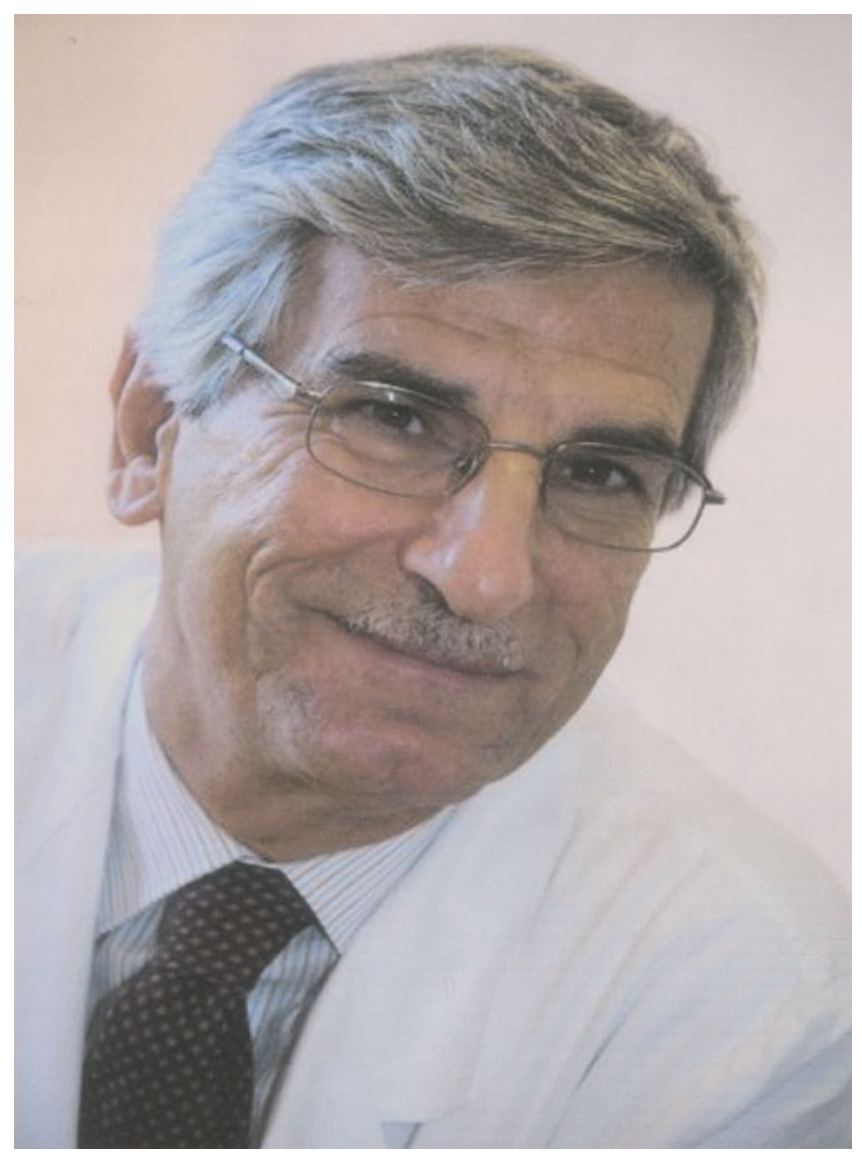

Fig. 1. Professor Carlo Bartolozzi 